\begin{tabular}{|c|l|}
\hline Title & Image restoration using the chiral Potts spin glass \\
\hline Author(s) & Carlucci, Domenico M.; Inoue, Jun-ichi \\
\hline Citation & $\begin{array}{l}\text { Physical Review E, 60/3), 2547-2553 } \\
\text { https://doi.org/10.1103/PhysRevE.60.2547 }\end{array}$ \\
\hline Issue Date & 1999-09 \\
\hline Doc URL & http://hdl.handle.net/2115/5808 \\
\hline Rights & Copyright $\odot 1999$ A merican Physical Society \\
\hline Type & article \\
\hline File Information & PRE60-3.pdf \\
\hline
\end{tabular}

Instructions for use 


\title{
Image restoration using the chiral Potts spin glass
}

\author{
Domenico M. Carlucci \\ Instituut voor Theoretische Fysica, K. U. Leuven, B-3001 Leuven, Belgium \\ Jun-ichi Inoue \\ Department of Physics, Tokyo Institute of Technology, Oh-okayama, Meguro-ku, Tokyo 152-8551, Japan
}

(Received 9 April 1999)

\begin{abstract}
We report on the image reconstruction (IR) problem by making use of the random chiral $q$-state Potts model, whose Hamiltonian possesses the same gauge invariance as the usual Ising spin glass model. We show that the pixel representation by means of the Potts variables is suitable for the gray-scale level image which cannot be represented by the Ising model. We find that the IR quality is highly improved by the presence of a glassy term, besides the usual ferromagnetic term under random external fields, as very recently pointed out by Nishimori and Wong. We give the exact solution of the infinite range model with $q=3$, the three-gray-scale-level case. In order to check our analytical result and the efficiency of our model, two-dimensional Monte Carlo simulations have been carried out on real-world pictures with three and eight gray-scale levels.

[S1063-651X(99)15008-6]

PACS number(s): 02.50.-r, 05.20.-y, 05.50.+q
\end{abstract}

\section{INTRODUCTION}

Recently, statistical mechanical approaches to the problems of information science have attracted a large amount of attention of researchers who are working in the field. Among these, particular interest has been given to techniques by which one tries to reconstruct an image from its corrupted version, e.g., sent by a defective fax, a fickle e-mail, etc. since any data transmission through a channel is in principle affected by some kind of noise. In the mathematical engineering fields, the traditional way to obtain the optimal recovered image has been regarded as a sort of optimization problem. In this framework, one first constructs the energy (cost) function so that this function represents the distance between the original image and the recovered one as properly as possible; then, one minimizes it using suitable heuristic methods like simulated annealing [1]. In fact, Geman and Geman [2] succeeded in constructing a method of image restoration using simulated annealing, and they discussed in detail the properties of its convergence including the optimal annealing schedule.

Successful results in this direction have been reached by means of the usual techniques of disordered spin systems, assuming that each spin is naturally associated to a pixel or bit. In language of the disordered spin systems, the optimization problems we just mentioned are naturally translated into a search of the ground state for a system possessing many local minima of order $\exp (N)$. In contrast, Marroquin et al. [3] found that the temperature of the system plays an important role for the image-recovering process. From the statistical mechanical point of view, each recovered image can be regarded as the equilibrium state of a random spin system. Marroquin et al. [3] investigated the effect of the temperature on the quality of image restoration by computer simulation and found the optimality of finite-temperature image restoration. Recently, this finite-temperature effect on image restoration was checked in a more careful way by Pryce and Bruce [4], although these works were restricted to numerical simulations. In the context of the convolutional error-correcting codes, Ruján [5] proposed finite-temperature decoding in which we regard the sign of the local magnetization at a specific temperature (this temperature is well known as the Nishimori temperature [6] in the field of spin glasses) as the correct bit. Recently Nishimori and Wong [7] pointed out that the optimal restoration of an image is also obtained at some specific temperature and showed that image restoration (IR) and error-correcting codes (ECC) theory can be treated within a single framework. Indeed, to the usual IR Hamiltonian, ferromagnetic, and random field terms, they added a spin-glass term borrowed from the ECC theory [8] used for a parity check. They could exactly solve the infiniterange spin model and find the optimal values of the temperature and field (referred to as hyperparameters from now on) at which the best retrieval quality is achieved. However, their works are restricted to the case of Ising spin systems and in this sense they are able to restore black-white pictures. On the other hand, there remain many open questions about the restoration of multicolor images or, somehow equivalently, gray-toned images.

This kind of problem has been also widely studied in the context of neural networks with multistate neurons, able to store and retrieve gray-scaled patterns (see [9] and references therein). For our purposes, we therefore map the set of the pixels onto $q$-state (chiral) Potts spins, with a ferromagnetic Hamiltonian in the presence of a random field (conventional IR) and, further on, a glass term (ECC-like term). The choice of the chiral Potts Hamiltonian is motivated by the fact that it exhibits the same gauge invariance as the Ising glass, although a work for the usual random Potts model is under consideration. Here, we show that, as in the Ising case [7], the presence of the glass term significantly increases the quality of the reconstructed image. We should mention that several remarkable studies about IR using the Potts model have been made by several authors. However, their works mostly depend on computer simulations. In addition, their methods (mean field annealing [10], cluster algorithm 
$[11,12]$, etc.) are devoted to restorations at zero temperature. Therefore, it seems that there exist a lot of open questions about IR using the Potts model, especially, about the performance of finite-temperature restoration.

In the next section, we will introduce our model within the image restoration theory and adopt the overlap as a measure of the restoration quality. In Sec. III, we will discuss the infinite-range model and give the exact expression for the overlap as a function of the temperature and external field, thus obtaining a relation between the temperature of source image and that of the restoration temperature. We shall also see an improvement of the restoration quality by adding the glassy term. Finally, in Sec. IV, guided by the infinite-range results, we will give explicit and realistic examples of image reconstructions for three and eight gray-scale pictures.

\section{MODEL AND IR FORMULATION}

As already mentioned in the Introduction, we choose to represent pixels of a gray-scaled image by means of $q$-component Potts spin variables. The usual Potts Hamiltonian $H=-\Sigma \delta_{\sigma_{i} \sigma_{j}}$ admits a complex representation [13] by means of the identity

$$
\delta_{\sigma_{i} \sigma_{j}}=\frac{1}{q} \sum_{r=0}^{q-1}\left(\sigma_{i}\right)^{r}\left(\sigma_{j}\right)^{q-r},
$$

where each spin takes on one of the $q$ roots of unity:

$$
\sigma_{i}=\exp \left(\frac{2 \pi i}{q} K_{i}\right) \quad\left(K_{i}=0, \ldots, q-1\right) .
$$

From now on, we will use the notation $\{\xi\}$ for the original pixels and $\{\sigma\}$ for the variables of the recovering process. Let us now send the original image through a noise channel not only by the form of $\xi_{i}^{r}$ itself but also by the following products $\xi_{i}^{r} \xi_{j}^{r *}=\xi_{i}^{r} \xi_{j}^{q-r}$. Without loss of generality we raised the spins and their products to some power $r$, since this corresponds only to a rotation in the complex circle. The reasons for this choice will be clear soon. For this expression, the output $\left(\left\{\tau^{(r)}\right\},\left\{J^{(r)}\right\}\right)$ is stochastically determined by the channel. For instance, in the case of a Gaussian channel (GC) the output function $P_{\text {out }}\left(\left\{J^{(r)}\right\},\left\{\tau^{(r)}\right\} \mid\{\xi\}\right)$ is given by

$$
\begin{aligned}
& P_{\text {out }}\left(\left\{J^{(r)}\right\},\left\{\tau^{(r)}\right\} \mid\{\xi\}\right) \\
&=\frac{1}{(2 \pi J)^{N_{B} / 2}} \frac{1}{(2 \pi \tau)^{N / 2}} \\
& \times \exp \left[-\frac{1}{2 J^{2}} \sum_{(i j)} \sum_{r=0}^{q-1}\left(J_{i j}^{(r)}-J_{0} \xi_{i}^{r} \xi_{j}^{q-r}\right)\right. \\
&\left.\times\left(J_{i j}^{(r) *}-J_{0} \xi_{i}^{q-r} \xi_{j}^{r}\right)\right] \\
& \quad \times \exp \left[-\frac{1}{2 \tau^{2}} \sum_{i} \sum_{r=0}^{q-1}\left(\tau_{i}^{(r)}-\tau_{0} \xi_{i}^{r}\right)\left(\tau_{i}^{(r) *}-\tau_{0} \xi_{i}^{q-r}\right)\right],
\end{aligned}
$$

where $J_{i j}^{(r)}$ and $\tau_{i}^{(r)}$ are complex numbers which satisfy

$$
\left(J_{i j}^{(r)}\right)^{*}=J_{i j}^{(q-r)}\left(\tau_{i}^{(r)}\right)^{*}=\tau_{i}^{(q-r)}
$$

in order to ensure the realness of the sums in Eq. (3).

Obviously, if a noise-free transmission could be achieved, we would obtain $\tau_{i}^{(r)}=\xi_{i}^{r}$ and $J_{i j}^{(r)}=\xi_{i}^{q-r} \xi_{j}^{r}$. The conditional probability $P\left(\{\sigma\} \mid\left\{J^{(r)}\right\},\left\{\tau^{(r)}\right\}\right)$, which is the probability that the source sequence is $\{\sigma\}$ provided that the outputs are $\{J\}$ and $\{\tau\}$, according to the Bayes theorem reads

$$
\begin{aligned}
P\left(\{\sigma\} \mid\left\{J^{(r)}\right\},\left\{\tau^{(r)}\right\}\right) \sim & \exp \left(\frac{\beta_{J}}{q} \sum_{(i j)} \sum_{r=1}^{q-1} J_{i j}^{(r)} \sigma_{i}^{(r)} \sigma_{j}^{(q-r)}\right. \\
& \left.+\frac{h}{q} \sum_{i} \sum_{r=1}^{q-1} \tau_{i}^{(r)} \sigma_{j}^{(q-r)}\right) P_{d}(\sigma),
\end{aligned}
$$

where $P_{d}(\sigma)$ is a model of the prior distribution $P_{s}(\xi)$, that is,

$$
P_{d}(\sigma) \equiv \exp \left(\frac{\beta_{d}}{q} \sum_{(i j)} \sum_{r=1}^{q-1} \sigma_{i}^{(r)} \sigma_{j}^{(q-r)}\right)
$$

Our choice of the above prior distribution (6) is due to the assumption that in the real world, images should be locally smooth. From this point of view, the distribution (6) is suitable because it gives a high probability if the nearestneighboring sites take the same value.

For the Ising model, in order to get the restored pixels out of the average quantities, the pixel at site $i$ (to be denoted as $\Sigma_{i}$ ) is naturally taken as the sign of the local magnetization. This means that the restored pixel is chosen as $\Sigma=+1$ ( $\Sigma$ $=-1$ ) if the spin points upward (downward) on average at the equilibrium. For our model, instead, since the value of the local magnetization is not simply confined to the interval $[-1,1]$, but runs all over the complex circle, we introduce the generalized restored variable

$$
\Sigma_{i}\left(\left\langle\sigma_{i}\right\rangle\right)=\exp \left[i \sum_{\alpha=0}^{q-1} \frac{2 \pi}{q} \alpha \Xi_{\alpha}\left(\theta_{i}\right)\right]
$$

with

$$
\Xi_{\alpha}(x)=\Theta\left(x-\frac{2 \pi}{q} \alpha+\frac{\pi}{q}\right)-\Theta\left(x-\frac{2 \pi}{q} \alpha-\frac{\pi}{q}\right),
$$

$\Theta$ being the usual step function, and

$$
\theta_{i}=\tan ^{-1}\left(\frac{\left\langle\operatorname{Re}\left[\sigma_{i}\right]\right\rangle}{\left\langle\operatorname{Im}\left[\sigma_{i}\right]\right\rangle}\right)
$$

In simpler words, $\Sigma_{i}$ is the closest spin on the circle to the value of the local magnetization $\left\langle\sigma_{i}\right\rangle \equiv\left\langle\operatorname{Re}\left[\sigma_{i}\right]\right\rangle$ $+i\left\langle\operatorname{Im}\left[\sigma_{i}\right]\right\rangle$. For $q=2$, it is straightforward to check that Eq. (7) reduces to a sign function up to a normalization constant. The quantities $\left\langle\operatorname{Re}\left[\sigma_{i}\right]\right\rangle$ and $\left\langle\operatorname{Im}\left[\sigma_{i}\right]\right\rangle$ are the average over the Boltzmann distribution $e^{-\mathcal{H}_{\text {eff }}}$ with the following effective Hamiltonian [14]: 


$$
\begin{aligned}
\mathcal{H}_{\mathrm{eff}} & -\frac{\beta_{J}}{q} \sum_{(i j)} \sum_{r=1}^{q-1} J_{i j}^{(r)}\left(\sigma_{i}\right)^{r}\left(\sigma_{j}\right)^{q-r} \\
& -\frac{\beta_{d}}{q} \sum_{(i j)} \sum_{r=1}^{q-1}\left(\sigma_{i}\right)^{r}\left(\sigma_{j}\right)^{q-r}-h \sum_{i} \sum_{r=1}^{q-1} \tau_{i}^{(r)} \sigma_{j}^{q-r} .
\end{aligned}
$$

Condition (4) gives the above Hamiltonian the same spin gauge symmetry as Ising spin glass, thus suppressing the spontaneous magnetization at low temperature which is present in the usual random Potts model. For the restoration purposes, the random field term aligns the spins according to the corrupted picture, whereas the ferromagnetic term ensures the smoothness, by suppressing the isolated pixels within one small cluster. Therefore, a balance between $\beta_{d}$ and $h$ will help us to reconstruct the original picture well. The first term, instead, has been recently introduced in the problem of image restoration by Nishimori and Wong [7] and this term has been well known as the parity check codes in the field of error-correcting codes. Obviously, this term carries much more information about the original picture than the other two terms. Therefore, the performance of the image recovery is expected to be improved by this term. As a measure of the restoration quality, we shall adopt the following overlap $M$ :

$$
\begin{aligned}
M & =\left[\frac{1}{q} \sum_{r=0}^{q-1} \xi_{i}^{q-r} \Sigma_{i}^{r}\right]_{\{\xi, J, \tau\}} \\
& \equiv \frac{1}{q} \sum_{r=0}^{q-1} \sum_{\xi} \sum_{J} \sum_{\tau} P_{\text {out }}\left(\left\{J^{(r)}\right\},\left\{\tau^{(r)}\right\} \mid\{\xi\}\right) P(\xi) \xi_{i}^{q-r} \sum_{i}^{r},
\end{aligned}
$$

in which (1/q) $\sum_{r=0}^{q-1} \xi_{i}^{q-r} \sum_{i}^{r}$ at each single site gives 1 if the original spin is in the same state as the restored one, and 0 otherwise. Here the dependence on the local magnetization is buried in the angle $\theta_{i}$, given by Eq. (9), and the sum over all the sites is understood. The main goal of this paper is to maximize the overlap $M$ as a function of the temperatures $\left(\beta_{J}\right.$ and $\beta_{d}$ ) and the external field $h$ (referred to as an estimate of the hyperparameters). In the next section, we will start with an exactly solvable model, that is, an infinite-range version of the Potts spin glass.

\section{MEAN FIELD SOLUTION}

We will now investigate the performance of our model within the mean field approximation; viz., each spin is influenced by all the others. As the source image, we will consider a ferromagnetic state generated by a Boltzmann distribution at some finite temperature $T_{s}$. For the sake of simplicity, we will restrict ourselves to the case of $q=3$, although the results can be generalized to any value of $q$. We thus assume that the original set of pixels $\{\xi\}$ is generated by a ferromagnetic three-state Potts Hamiltonian with probability

$$
P(\xi)=\frac{1}{\mathcal{Z}_{s}\left(\beta_{s}\right)} \exp \left[\frac{\beta_{s}}{2 N} \sum_{i<j}\left(\xi_{i} \xi_{j}^{*}+\xi_{i}^{*} \xi_{j}\right)\right],
$$

where $\mathcal{Z}_{s}\left(\beta_{s}\right)$ is a normalization constant and $\beta_{s}$ is the inverse source temperature. According to the conditional probability, the observables are computed as

$$
\begin{aligned}
{[\langle f\rangle]_{\{\xi, J, \tau\}}=} & \sum_{\xi} \sum_{J} \sum_{\tau} P\left(\left\{J^{(r)}\right\},\left\{\tau^{(r)}\right\} \mid\{\xi\}\right) \\
& \times P(\xi) \frac{\operatorname{Tr}_{\sigma} f \mathrm{e}^{-\mathcal{H}_{\mathrm{eff}}}}{\mathcal{Z}},
\end{aligned}
$$

with

$$
\mathcal{Z} \equiv \operatorname{Tr}_{\sigma} \exp \left(-\mathcal{H}_{\text {eff }}\right) .
$$

It is rather straightforward to average out the disorder by means of the well-known replica trick [16] and, assuming a replica symmetry ansatz and isotropy (no dependence on $r$ ), the saddle point equations for the order parameters are given by

$$
\begin{aligned}
& {\left[\left\langle\sigma_{i}^{r}\right\rangle\right] \equiv m=\frac{1}{\mathcal{Z}_{s}} \sum_{\xi} e^{\beta_{s}\left(m_{s}^{(1)} \operatorname{Re}[\xi]+m_{s}^{(2)} \operatorname{Im}[\xi]\right)}} \\
& \times \int \frac{d u}{\sqrt{\pi}} \frac{d \mathrm{v}}{\sqrt{\pi}} e^{-u^{2}-\mathrm{v}^{2}} \frac{Z_{\mathrm{cos}}(\xi)}{Z(\xi)}, \\
& {\left[\operatorname{Re}\left[\xi_{i}\right]\left\langle\sigma_{i}^{r}\right\rangle\right] \equiv t_{1}=\frac{1}{\mathcal{Z}_{s}} \sum_{\xi} \operatorname{Re}[\xi] e^{\beta_{s}\left(m_{s}^{(1)} \operatorname{Re}[\xi]+m_{s}^{(2)} \operatorname{Im}[\xi]\right)}} \\
& \times \int \frac{d u}{\sqrt{\pi}} \frac{d \mathrm{v}}{\sqrt{\pi}} e^{-u^{2}-\mathrm{v}^{2}} \frac{Z_{\mathrm{cos}}(\xi)}{Z(\xi)}, \\
& {\left[\operatorname{Im}\left[\xi_{i}\right]\left\langle\sigma_{i}^{r}\right\rangle\right] \equiv t_{2}=\frac{1}{\mathcal{Z}_{s}} \sum_{\xi} \operatorname{Im}[\xi] e^{\beta_{s}\left(m_{s}^{(1)} \operatorname{Re}[\xi]+m_{s}^{(2)} \operatorname{Im}[\xi]\right)}} \\
& \times \int \frac{d u}{\sqrt{\pi}} \frac{d \mathrm{v}}{\sqrt{\pi}} \mathrm{e}^{-u^{2}-\mathrm{v}^{2}} \frac{Z_{\cos }(\xi)}{Z(\xi)}, \\
& {\left[\left\langle\sigma_{i}^{r}\right\rangle\left\langle\sigma_{j}^{q-r}\right\rangle\right] \equiv Q=\frac{1}{\mathcal{Z}_{s}} \sum_{\xi} \mathrm{e}^{\beta_{s}\left(m_{s}^{(1)} \operatorname{Re}[\xi]+m_{s}^{(2)} \operatorname{Im}[\xi]\right)}} \\
& \times \int \frac{d u}{\sqrt{\pi}} \frac{d \mathrm{v}}{\sqrt{\pi}} \mathrm{e}^{-u^{2}-\mathrm{v}^{2}} \frac{1}{Z^{2}(\xi)}\left[Z_{\cos }^{2}(\xi)\right. \\
& \left.+Z_{\sin }^{2}(\xi)\right] \text {. }
\end{aligned}
$$

Here $m_{s}^{(1)}$ and $m_{s}^{(2)}$ are simply the real and imaginary components of the source magnetization, viz., the usual nonrandom Potts model [13] mean field equations

$$
\begin{aligned}
{\left[\operatorname{Re}\left[\xi_{i}\right]\right] \equiv } & m_{s}^{(1)}=\frac{1}{\mathcal{Z}_{s}}\left(e^{\beta_{s} m_{s}^{(1)}}-e^{-\beta_{s} m_{s}^{(1) / 2}}\right. \\
& \left.\times \cosh \left[(\sqrt{3} / 2) \beta_{s} m_{s}^{(2)}\right]\right) \\
{\left[\operatorname{Im}\left[\xi_{i}\right]\right] \equiv m_{s}^{(2)}=} & \frac{1}{\mathcal{Z}_{s}} \sqrt{3} e^{-\beta_{s} m_{s}^{(1) / 2}} \sinh \left[(\sqrt{3} / 2) \beta_{s} m_{s}^{(2)}\right]
\end{aligned}
$$

and 


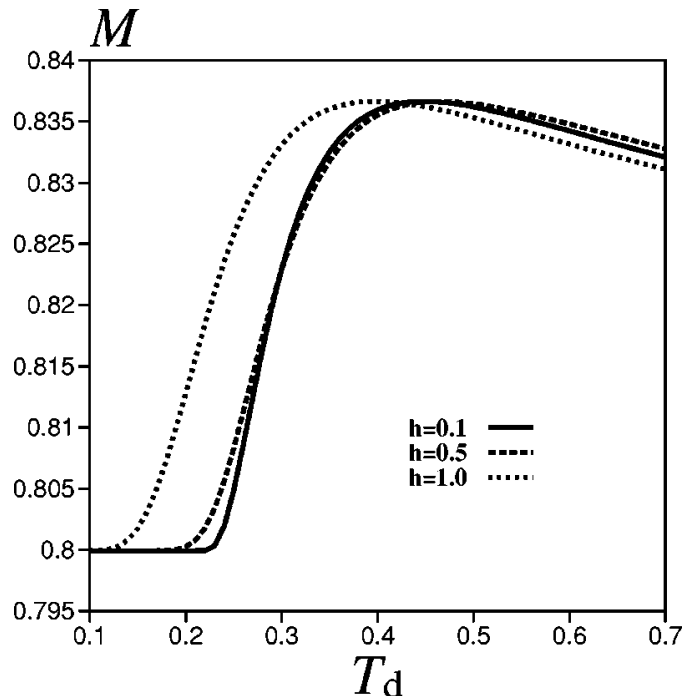

FIG. 1. Overlap $M$ as a function of $T_{d}$ for different values of $h$. The maximum value $M_{\max }$ does not depend on $h$.

$$
\begin{gathered}
\mathcal{Z}_{s}=e^{\beta_{s} m_{s}^{(1)}}+2 e^{-\beta_{s} m_{s}^{(1) / 2}} \cosh \left[(\sqrt{3} / 2) \beta_{s} m_{s}^{(2)}\right] \\
Z_{\cos }(\xi)=e^{U(\xi)}-e^{-U(\xi) / 2} \cosh V \\
Z_{\sin }(\xi)=\sqrt{3} e^{-U(\xi) / 2} \sinh V \\
Z(\xi)=e^{U(\xi)}+2 e^{-U(\xi) / 2} \cosh V
\end{gathered}
$$

with

$$
\begin{gathered}
U=u\left[\frac{\beta_{J}^{2} J^{2}}{q^{2}} Q+\tau^{2} h^{2}\right]^{1 / 2}+\frac{\beta_{d}}{q} m+\frac{\beta_{J} J_{0}}{q}\left[t_{1} \operatorname{Re}[\xi]+t_{2} \operatorname{Im}[\xi]\right] \\
+\tau_{0} h \operatorname{Re}[\xi], \\
V=\frac{\sqrt{3}}{2} \frac{\beta_{J} J}{q} Q^{1 / 2} \mathrm{v} .
\end{gathered}
$$

Finally, the overlap $M$ is expressed as the weighted average

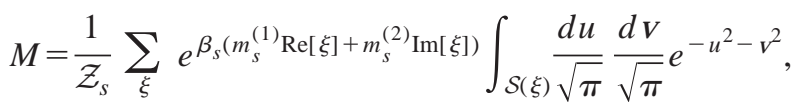

receiving contributions from the following $q=3$ regions in the complex circle:

$$
\begin{aligned}
\mathcal{S}(1)= & \left\{u, \mathrm{v} \mid-\frac{\pi}{3} \leqslant \tan ^{-1} \frac{Z_{\text {sin }}}{Z_{\cos }} \leqslant \frac{\pi}{3} \cap Z_{\cos }>0\right\}, \\
\mathcal{S}\left(e^{2 \pi i / 3}\right)= & \left\{u, \mathrm{v} \mid\left(\frac{\pi}{3} \leqslant \tan ^{-1} \frac{Z_{\sin }}{Z_{\cos }} \leqslant \frac{\pi}{2} \cap Z_{\cos } \geqslant 0, Z_{\sin }>0\right)\right. \\
& \left.\cup\left(-\frac{\pi}{2} \leqslant \tan ^{-1} \frac{Z_{\text {sin }}}{Z_{\cos }} \leqslant 0 \cap Z_{\cos } \leqslant 0, Z_{\sin } \geqslant 0\right)\right\},
\end{aligned}
$$

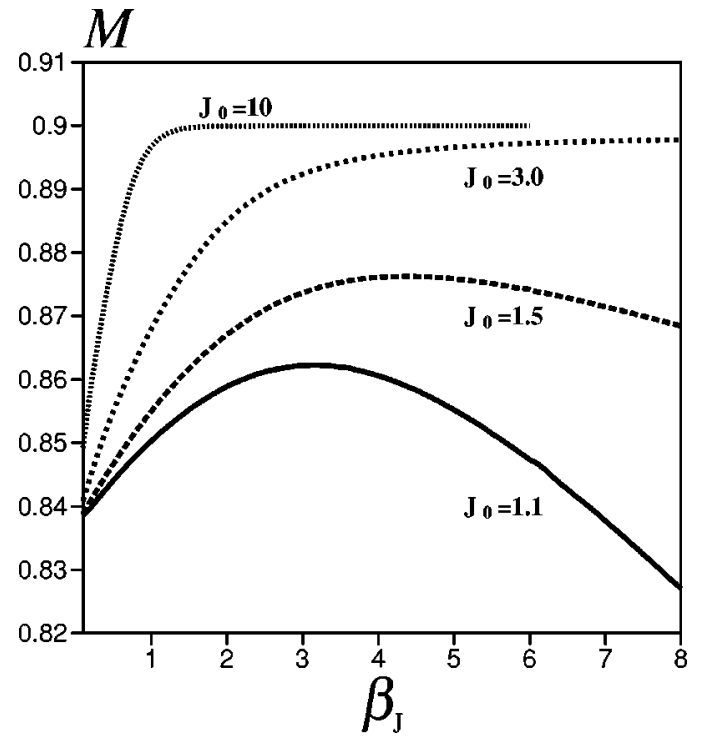

FIG. 2. Overlap $M_{\max }$ as a function of the exchange temperature $\beta_{J}$ for several values of $J_{0}$. The overlap improves even for small values of the exchange term.

$$
\begin{aligned}
\mathcal{S}\left(e^{4 \pi i / 3}\right)= & \left\{u, \mathrm{v} \mid\left(\frac{\pi}{2} \leqslant \tan ^{-1} \frac{Z_{\text {sin }}}{Z_{\cos }} \leqslant-\frac{\pi}{3} \cap Z_{\cos }>0, Z_{\sin } \geqslant 0\right)\right. \\
& \left.\cup\left(0 \leqslant \tan ^{-1} \frac{Z_{\text {sin }}}{Z_{\text {cos }}} \leqslant \frac{\pi}{2} \cap Z_{\cos }<0, Z_{\sin } \leqslant 0\right)\right\} .
\end{aligned}
$$

We first assume that the exchange term is absent $\left(\beta_{J}=0\right)$ [15]; that is, no redundancy is fed into the channel. In this case, the saddle point equations (15)-(18) are drastically simplified and the overlap (23) simply reads

$$
\begin{aligned}
M= & \frac{\mathrm{e}^{\beta_{s} m_{s}}}{\mathcal{Z}_{s}} \operatorname{Erf}\left[-\sqrt{2} \frac{\beta_{d} m+\tau_{0} h}{\tau h}\right] \\
& +\frac{e^{-1 / 2}}{\mathcal{Z}_{s}}\left\{1-\operatorname{Erf}\left[-\sqrt{2} \frac{\beta_{d} m-\tau_{0} h}{\tau h}\right]\right\},
\end{aligned}
$$

with the magnetization given by

$$
\begin{aligned}
m= & \frac{e^{\beta_{s} m_{s}}}{\mathcal{Z}_{s}} \int \frac{d u}{\sqrt{\pi}} e^{-u^{2}} \\
& \times \frac{1-\exp \left[-\frac{3}{2}\left(u \tau h+\beta_{d} m / q+\tau_{0} h\right)\right]}{1+2 \exp \left[-\frac{3}{2}\left(u \tau h+\beta_{d} m / q+\tau_{0} h\right)\right]} \\
& +\frac{2 e^{-\beta_{s} m_{s} / 2}}{\mathcal{Z}_{s}} \int \frac{d u}{\sqrt{\pi}} e^{-u^{2}} \\
& \times \frac{1-\exp \left[-\frac{3}{2}\left(u \tau h+\beta_{d} m / q-\tau_{0} h / 2\right)\right]}{1+2 \exp \left[-\frac{3}{2}\left(u \tau h+\beta_{d} m / q-\tau_{0} h / 2\right)\right]},
\end{aligned}
$$

where we defined $\operatorname{Erf}(x) \equiv \int_{x}^{\infty} \mathrm{e}^{-x^{2}} d x / \sqrt{\pi}$.

The problem is thus reduced to a one-dimensional model, corresponding to an Ising model in which the length of spin turns out to be $(+1,-1 / 2)$ instead of $(+1,-1)$. This is not 

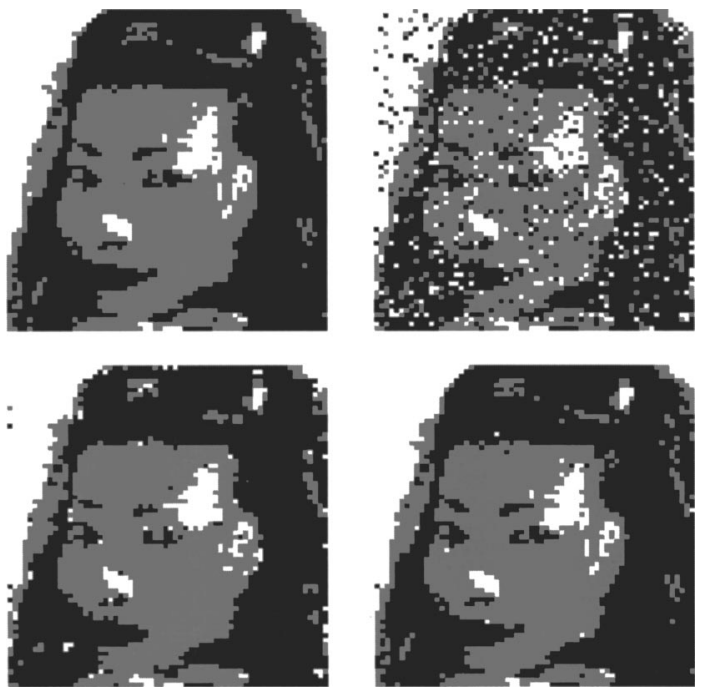

FIG. 3. Upper left: original three-gray-scale-level image. Upper right: $15 \%$ of noise. Lower left: restoration without exchange term. Lower right: restoration with exchange term

surprising if one thinks that the fluctuations along the imaginary axis are governed only by the glassy term; meanwhile, the magnetic field acts along the real direction. In Fig. 1, we plotted the overlap $M$ as a function of $T_{d}$ for the some values of $h$. It is straightforward to check that the maximum value of the overlap $M_{\max }$ does not depend on magnetic field $h$, since at the stationary point $\left(\partial M / \partial \beta_{d}=0\right) \mathrm{m}$ is proportional to the magnetic field

$$
\frac{1}{2} \beta_{s} m_{s}=\frac{1}{3} m \beta_{d} \frac{\tau_{0}}{\tau^{2} h}+\frac{1}{4} \frac{\tau_{0}^{2}}{\tau^{2}}
$$

This feature holds also for the Ising case, although the stationary equation (27) is simpler:

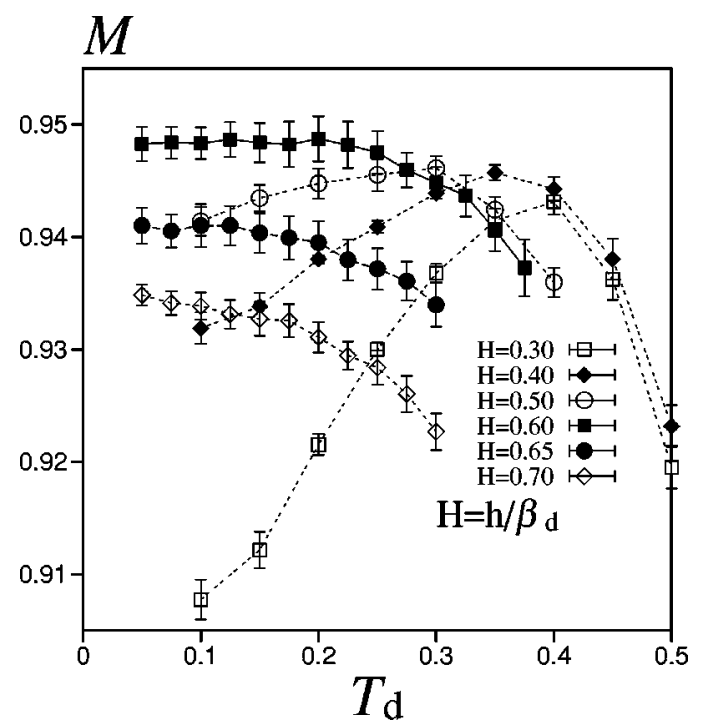

FIG. 4. Overlap $M$ as a function of the decoding temperature $T_{d}$ at $\beta_{J}=0$ (left). The system size is $64 \times 72$ and each line is averaged over four different samples.

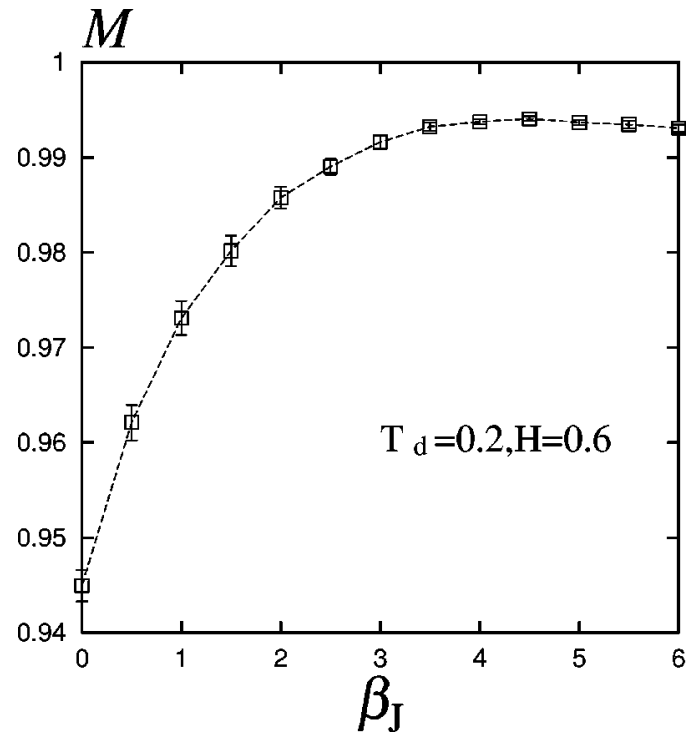

FIG. 5. Overlap as a function of $\beta_{J}$. We set the parameters $\left(H, T_{d}\right)=(0.6,0.2)$ which gives the maximum in the absence of the exchange term.

$$
\beta_{s} m_{s}=m \beta_{d} \frac{\tau_{0}}{\tau^{2} h}
$$

Expression (27) is thought to be valid only for the infiniterange model, as confirmed in the next section by numerical results in $d=2$.

Now we set the decoding temperature at the optimal value, that is, $M\left(T_{d}^{\text {opt }}\right) \equiv M_{\max }$, and we switch the exchange interaction $\left(\beta_{J} \neq 0\right)$ as depicted in Fig. 2. We notice that also a small amount of redundancy highly improves the value of the overlap $M_{\max }$ which quickly increases and slowly decreases, after the peak; meanwhile, the exchange term becomes dominant to the ferromagnetic one.
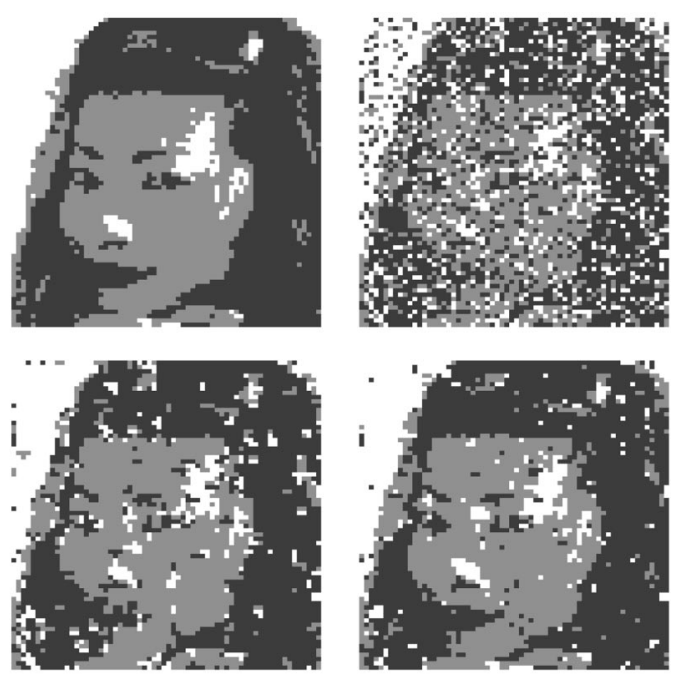

FIG. 6. Upper left: original three-gray-scale-level image. Upper right: $30 \%$ of noise. Lower left: restoration without exchange term. Lower right: restoration with exchange term. 

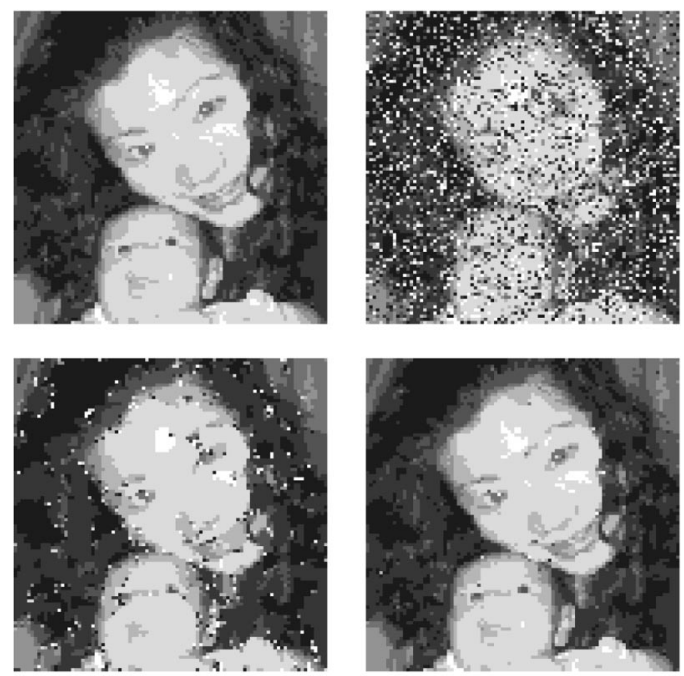

FIG. 7. Upper left: original eight-gray-scale-level image. Upper right: $20 \%$ of noise. Lower left: restoration without exchange term. Lower right: restoration with exchange term.

\section{MONTE CARLO SIMULATIONS FOR REAL-WORLD PICTURES}

Although for mere restoration aims it is not wise to smoothen two points far away from each other, we shall see that the infinite-range model provides a useful guide for the more interesting case of real-world pictures, since the results remain qualitatively similar. We thus carried out Monte Carlo simulations for realistic pictures with a short-range effective Hamiltonian. In this case, the ferromagnetic term will be concerned only with points within the range of interaction and two points far away will not influence each other. It would be extremely interesting to study the restoration quality as a function of the interaction radius, but this goes beyond the aim of the present work and we limit ourselves to a first-nearest-neighbor interaction Hamiltonian. Therefore let us consider a simple $q=3$ gray-scale-level picture (upper left
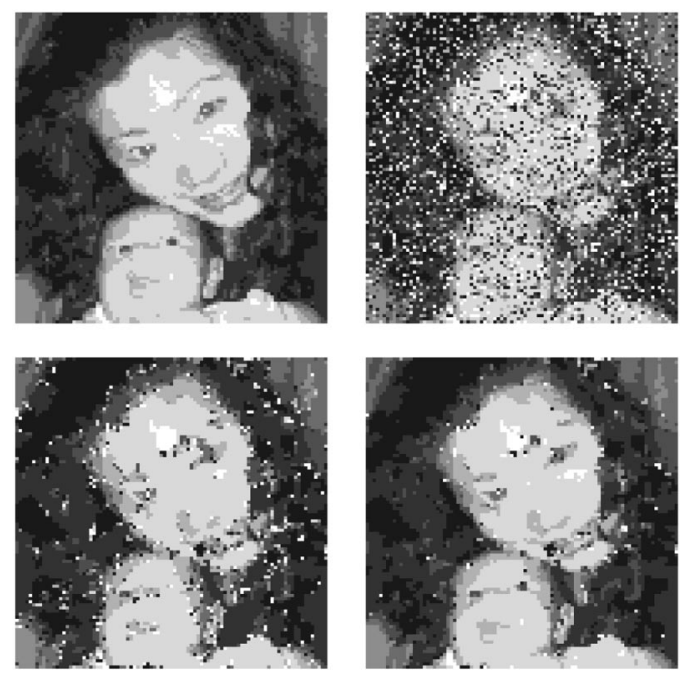

FIG. 8. Upper left: original eight-gray-scale-level image. Upper right: $30 \%$ of noise. Lower left: restoration without exchange term. Lower right: restoration with exchange term.

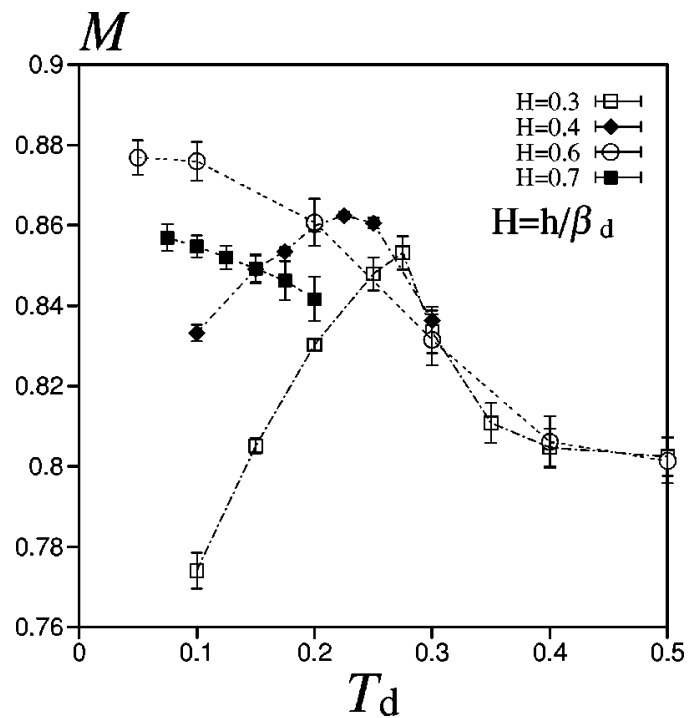

FIG. 9. Overlap $M$ as a function of the decoding temperature $T_{d}$ at $\beta_{J}=0$ (left). The system size is $93 \times 100$ and each line is averaged over four different samples.

of Fig. 3), where each pixel has been randomly flipped to another value with some probability, say, $p=0.15$ (upper right of Fig. 3). The curves shown in Fig. 4 are the result of the restoration process without the glassy term, that is, $\beta_{J}$ $=0$, at different values of the ratio $H=h / \beta_{d}$. Here the maximum value of the overlap is achieved around $H_{\max } \equiv h / \beta_{d}$ $\sim 0.6$ and $T_{d} \sim 0.2$ and the corresponding restored image is drawn in the lower left of Fig. 3. Adding the glassy term at $H_{\text {max }}$ fixed improves drastically the value of the overlap and the quality of the restored image (lower right of Fig. 3), drawn at the peak of Fig. 5. The same procedure is repeated in the presence of higher noise, $p=0.30$, at the same $H_{\max }$ and $\beta_{J ; \max }$ and the results of the restoration are shown in Figs. 6. Finally, we applied the same algorithm to an eightgray-scale-level picture with $20 \%$ and $30 \%$ of noise, upper images in Figs. 7 and 8. The results without exchange term

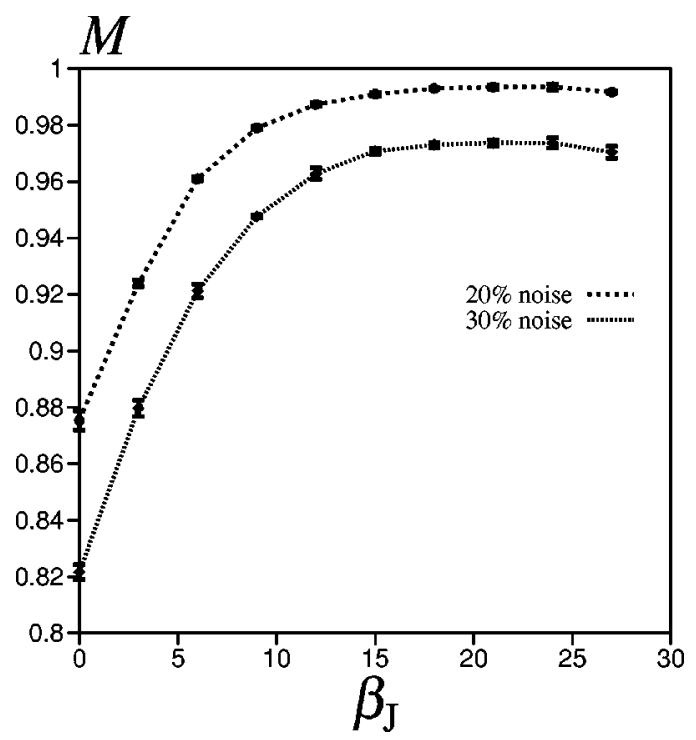

FIG. 10. Overlap as a function of $\beta_{J}$. We set the parameters $\left(H, T_{d}\right)=(0.6,0.1)$ which gives the maximum in the absence of the exchange term. 
are shown in shown Fig. 9. Once again we find a maximum for some values of $T_{d}$ and $H$ and the corresponding restored images are shown in Fig. 10.

\section{CONCLUSIONS}

In this paper, we investigated the possibility of grayscaled image restoration using the chiral random Potts model. We solved exactly the infinite-range version, thus deriving an explicit expression of the overlap as a function of the estimates of the hyperparameters $h, \beta_{d}$, and $\beta_{J}$. In the absence of the glassy term, we obtained an exact relation between the restoration temperature $\beta_{d}$ and the source temperature $\beta_{s}$ which gives the maximum value of the overlap. This seems a highly nontrivial result because it is natural for us to assume that the best recovery of the image should be achieved for $\beta_{d}=\beta_{s}$, as it turns out to be true for the Ising case [7]. The Monte Carlo results on real pictures confirmed the expected high improvement due to the presence of the redundancy, i.e., the glassy term. However, so far, in our prescription to recover a corrupted image at the best restoration values, one is supposed to know the original data. In other words, the receiver has to meet the sender at least once to find the optimal restoration values. Only after that will the other receivers be able to get an optimal restoration for the same image, provided that the channels remain, at least qualitatively, unchanged. In this sense, it would be extremely useful to provide some a priori criteria (the receiver will not be supposed to meet the sender) for the optimum values of the hyperparameters, once that some intrinsic characteristics (e.g., temperature) of the original image are known. Therefore, in order to check if relation (27) still holds down to two dimensions, we restored $q=3$ ferromagnetic snapshots generated at some known temperature. However, so far we have not yet obtained reliable results and detailed investigations in this direction will be reported in a forthcoming paper.

\section{ACKNOWLEDGMENTS}

We thank Professor Hidetoshi Nishimori for useful discussions and showing us his paper prior to publication. We also thank Professor Kazuyuki Tanaka for useful advice and fruitful discussions. The authors were supported by JSPSRoyal Society/British Council Anglo-Japanese Scientific Cooperation Program. One of the authors (D.M.C.) started this work under JSPS Grant No. P96215.
[1] S. Kirkpatrick, C. D. Gelatt, and M. P. Vecchi, Science 220, 671 (1983).

[2] S. Geman and D. Geman, IEEE Trans. Pattern. Anal. Mach. Intell. 6, 721 (1984).

[3] J. Marroquin, S. Mitter, and T. Poggio, J. Am. Stat. Assoc. 82, 76 (1987).

[4] J. M. Pryce and A. D. Bruce, J. Phys. A 28, 511 (1995).

[5] P. Ruján, Phys. Rev. Lett. 70, 2968 (1993).

[6] H. Nishimori, Prog. Theor. Phys. 66, 1169 (1981).

[7] H. Nishimori and K. Y. M. Wong (unpublished).

[8] N. Sourlas, Nature (London) 339, 693 (1989).

[9] D. Bollé, B. Vinck, and V. A. Zagrebnov, J. Stat. Phys. 70, 1099 (1993).
[10] J. Zhang, IEEE Trans. Signal Process. 40, 2570 (1992).

[11] K. Tanaka and T. Morita, in Theory and Applications of the Cluster Variation and Path Probability Methods, edited by J. L. Morán-López and J. M. Sanches (Plenum Press, New York, 1996).

[12] T. Morita and K. Tanaka, Pattern Recogn. Lett, 18, 1479 (1997).

[13] L. Mittag and M. J. Stephen, J. Phys. A 7, L109 (1974).

[14] H. Nishimori and M. J. Stephen, Phys. Rev. B 27, 5644 (1983).

[15] H. Nishimori, Phys. Rev. B 28, 4011 (1983).

[16] D. Sherrington and S. Kirkpatrick, Phys. Rev. Lett. 35, 1792 (1975). 